\title{
Review: simplifying dosing regimens appears to improve treatment adherence in patients with high blood pressure in ambulatory settings
}

Schroeder K, Fahey T, Ebrahim S. Interventions for improving adherence to treatment in patients with high blood pressure in ambulatory settings. Cochrane Database Syst Rev 2004;(2):CD004804.

\section{Q In patients with high blood pressure in ambulatory settings, what is the effectiveness of interventions aimed at increasing adherence to blood pressure lowering medication?}

\section{METHODS}

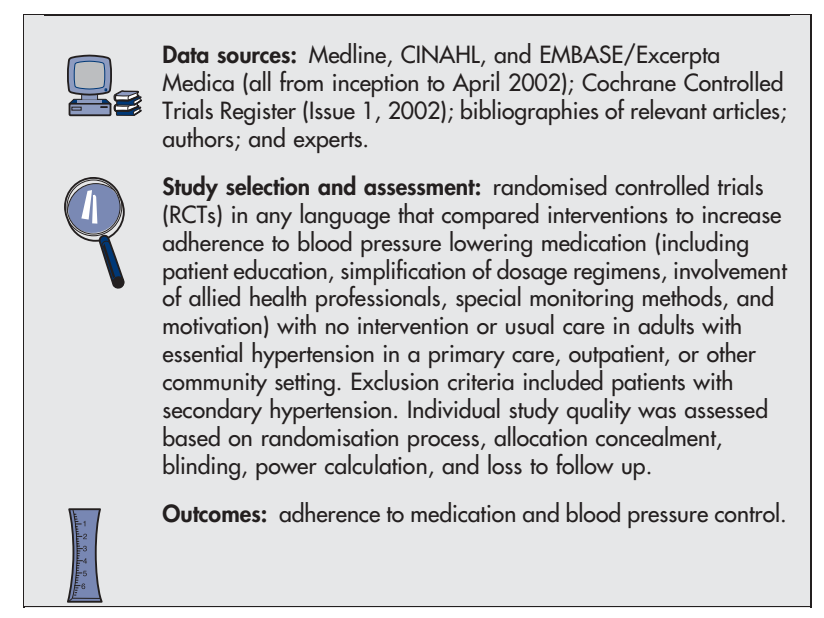

\section{MAIN RESULTS}

38 RCTs (15 519 patients) of 58 different interventions met the selection criteria. Follow up duration ranged from 2-60 months. Adherence was measured in various ways including self report, direct questioning, pill counts, and the Medication Event Monitoring System. Results were not pooled because of heterogeneity among studies. 19 RCTs reported an improvement in adherence alone, of which 13 also reported blood pressure changes. 7 RCTs reported an improvement in adherence combined with a reduction in blood pressure. 7 RCTs reported a reduction in blood pressure without an increase in adherence. 15 RCTs did not report a blood pressure outcome. None of the studies examined major clinical endpoints. Of 9 RCTs that used simplification of dosing regimens, 7 reported improved adherence; relative increase in adherence ranged from 8$20 \%$. Of 6 studies that used patient education, only l small RCT reported improved adherence with no effect on blood pressure. Of 24 RCTs that used patient motivation, support, and reminders, 10 reported mostly small increases in adherence to a maximum of $23 \%$. Of 18 RCTs that used complex combined health and organisational interventions, 8 reported increased adherence ranging from 5-41\%.

For correspondence: Dr $\mathrm{K}$ Schroeder, Division of Primary Health Care University of Bristol, Bristol, UK. k.schroeder@bristol.ac.uk

Source of funding: UK National Health Service Executive South and West Research and Development Fund.

\section{CONCLUSIONS}

In patients with high blood pressure in ambulatory settings, simplifying dosing regimens appears to increase adherence to blood pressure lowering medication. However, effects on subsequent blood pressure are unclear. Motivational strategies and complex interventions may improve treatment adherence, but more evidence is needed. Patient education alone does not appear to improve treatment adherence.

\section{Commentary}

7 he review by Schroeder et al assesses the effects of strategies for improving adherence to medication and blood pressure control (including simplifying dosing regimens) in patients with hypertension. The findings will be of interest to nurses in ambulatory settings who care for patients on oral antihypertensive medications.

The data sources and study selection process enhance the likelihood that the review includes the most relevant studies. Exclusion criteria limit the generalisability of the results to ambulatory settings and patients with primary hypertension. The review did not examine drug interactions, side effects, or costs in relation to level of adherence or level of blood pressure control.

The authors conclude that in ambulatory settings, simplifying dosing regimens increases adherence to blood pressure lowering medication in patients with high blood pressure but that the effect on subsequent blood pressure levels is unclear. More evidence is required to determine if motivational strategies and complex interventions are as effective for improving treatment adherence. The finding that patient education alone does not promote adherence has a direct impact on nursing practice. Nurses will need to combine educational interventions with other strategies and continue to assess the effects of their approaches on both adherence and blood pressure levels.

Treatment with antihypertensive medication is an important component of multi-interventional blood pressure management and cardiovascular risk reduction care plans. ${ }^{12}$ The review by Schroeder et al strengthens the position that simplifying antihypertensive dosing regimens can lead to improved medication adherence in patients with primary hypertension who access care through ambulatory settings. Michelle Clifford-Middel, RN-EC, BScN, PHC NP IOOF Senior Citizen Homes Incorporated Barrie, Ontario, Canada

1 Dyer AR, Liu K, Walsh M, et al. Ten-year incidence of elevated blood pressure and its predictors: the CARDIA study. Coronary Artery Risk Development in (Young) Adults. J Hum Hypertens 1999;13:13-21.

2 The sixth report of the Joint National Committee on Prevention, Detection, Evaluation, and Treatment of High Blood Pressure. Arch Intern Med 1997;157:2413-46. 